\title{
Using HSPA to improve the telemedical links on a moving ambulance
}

\author{
Konstantinos Banitsas ${ }^{1}$, Sapal Tachakra ${ }^{2}$, Euclid Stefanidis ${ }^{1}$, Konstantinos Boletis ${ }^{1}$ \\ ${ }^{1}$ School of Engineering and Design, E\&CE, Brunel University, West London, England \\ ${ }^{2}$ A\&E department, North West London Hospitals, U.K.
}

\begin{abstract}
As the demand for faster and more effective health care increases, there is a growing need to establish mobile, high-speed communications between a moving ambulance and a consultation point (usually a hospital). The recent addition of HSPA (HSDPA and HSUPA) into the UMTS suite provides higher bandwidth and reduced delays, making this choice ideal for real-time telemedical applications.

In this paper, we will describe a set of scenarios that took place in a typical large city area, along with their equivalent results: a moving ambulance was linked with a consultation station using HSPA and several videoconferencing sessions were initiated. Best-case, worst-case and average scenarios were recorded. Furthermore, in areas where the UMTS reception was marginal, a repeater was placed on top of the vehicle to boost up the signal power and thus maintain the higher bandwidth. Finally, treating doctors were asked to evaluate the effectiveness of this system's outputs, based on a variety of objective and subjective criteria.
\end{abstract}

Keywords - HSPA, HSDPA, HSUPA, ambulance, telemedicine, 3G

\section{INTRODUCTION}

A MBULANCES nowadays become increasingly equipped with state of the art technology capable of handling a variety of medical issues. The objective should not only be to handle emergencies and first aid, but also to provide the patient with health services that would only been possible when admitted to the hospital.

A good example of the current situation is the ambulance service of the National Health care provider of UK (NHS). It is reported [1] that over the course of last year, 1.3 million emergency calls were received. From those, more than a quarter were classified as category A (most urgent). On the above figure, $87 \%$ of the patients were delivered in time to the nearest hospital (having an average trip time of about 12 minutes) while the remaining $13 \%$ was delayed due to a variety of reasons, mainly to do with traffic congestion. There is a clear need for more immediate treatment (as well as advanced diagnosis in cases that the patient needs treatment like thrombolysis, resuscitation, etc) for these patients; something that should start from the moment they enter the ambulance and would involve high quality links from the moving ambulance, to a diagnostic and treatment center (usually a hospital).

Several attempts were made in the past to create such links. Some of them used GSM communication to transmit the patient's physiological signals (heart rate, blood pressure, breathing rate, etc) [2]. These suffered by the low data rate of GSM and the inability to support any other related multimedia services. Other attempts used GPRS to transfer still images of the patients being carried by the ambulance [3]. Finally, and with the widespread use of UMTS (3G) telephony, downlink rates of 128 to $384 \mathrm{kbps}$ were available to mobile users. These links were utilized to initiate videoconferencing sessions between a moving ambulance and the hospital so a doctor or consultant can deliver an expert opinion while the patient was carried to the hospital [3], [4].

During the last year, some additions to the UMTS suite were rectified that allowed for an improved data rate of UMTS: High Speed Download Packet Access (HSDPA) and High Speed Upload Packet Access (HSUPA). Both constitute the High Speed Packet Access (HSPA) suite, an improvement to the $3 \mathrm{G}$ system that is rightfully referred to as $3.5 \mathrm{G}$ [5]

HSDPA and HSUPA were standardized as part of the $3 \mathrm{G}$ Partnership Project (3GPP) release 5 and 6 respectively. HSDPA peak download rate initially available to the user elements (UE) was 1.8 Mbps but soon was increased to 3.6, 7.2 and currently to $14.4 \mathrm{Mbps}$, and is planned to reach several tens of Mbps in the near future [6]. HSUPA, on the other hand, allows for asymmetric upload data rates of 5.76 Mbps while having a reduced download rate. Some recent research focused only on the use of HSDPA to carry realtime biosignals (ECG, NIBP, blood glucose, $\mathrm{SpO}_{2}$ and hart rate) [7] and low rate/low resolution video [8] from within a moving ambulance. However, the emphasis of those systems was in the biosignals, which only accounted for a fraction of the bandwidth needed for high-resolution videoconferencing and as such, they placed much less demand on the communication channel.

Both the above enhancements introduce several new channels into the lower ISO layers. As a result, the HSPA suite manages to deliver at least a $3 \mathrm{x}$ increase in sector, $6 \mathrm{x}$ increase in user throughput and a $4 x$ increase in the number of users, compared with the UMTS release-99. It also reduces network latency below $100 \mathrm{~ms}$ (typical is $75 \mathrm{~ms}$ ) by using shorter time transmission intervals (TTI) of $2 \mathrm{~ms}$ and thus significantly improving TCP/IP services and increasing the overall QoS [9].

By now, it should be clear that compared with the conventional UMTS, HSPA suite is a significant enhancement for real-time multimedia services that demand high data rate and low latencies (round trip delays). This serves as an ideal tool for applications like videoconferencing from within a moving ambulance. 


\section{Methodology}

We focus on the use of HSPA to transfer live video, sound and high-resolution images from within a moving ambulance, to a consultation point, while using Internet links. The city chosen for this experiment is Thessaloniki, at north Greece. The wider area of Thessaloniki (second largest city of Greece) has a population of over a million and is a city notorious for its heavy traffic conditions. Despite the relatively small size of the city, ambulance transportations often take more than 20 minutes while navigating the congested city streets risking the lives of critically ill or injured patients in desperate need of medical treatment. As Thessaloniki's city center is completely covered by HSPA, it serves as an ideal example of any large city area.

We placed a laptop computer (HP N610c) inside the ambulance and connected it to either a webcam (Logitec QuickCam E3500) or to a high-quality video camera (Sony PC110E), with the latter performing significantly better. The operator (paramedic) used the camera to point to either the patient injuries or to any of the screens displaying the patient's physiological condition (e.g. ECG). We developed a videoconferencing software running on the laptop that picks up these images videos and sounds and transmits them to the modem. We used both an HSDA mobile phone (Sony Ericsson W910) capable of acting as a modem and a USB dongle specifically manufactured for HSPA access over a laptop (Globe Surfer iCON 7.2 S). High-quality images were sent to the consultation point in the hospital, using the same software. The paramedics had to follow the advice of the treating doctor in the hospital and take action in cases where they did not have the expertise to do so. Two-way videoconferencing took place so that the patient had an increased feeling of security and the paramedic were assisted with visual aids [Fig. 1].

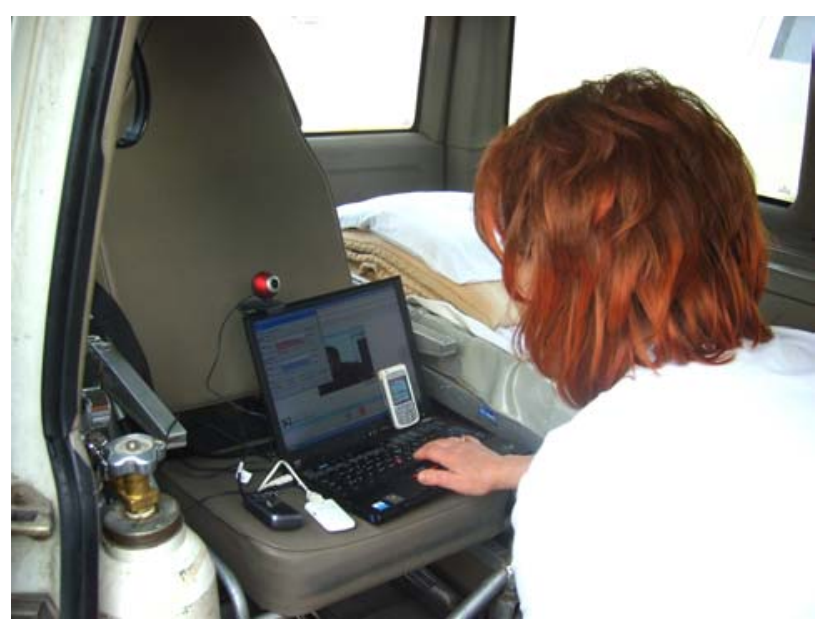

Figure 1. Using HSPA links to transfer high quality video to the hospital

For four days an ambulance followed a wide range of routes that would result in a) the best-case communication links, b) no HSPA communication links (fall back to $3 \mathrm{G}$ ), c) high-traffic congested links and d) finally no $3 \mathrm{G}$ coverage (fall back to GPRS). The ambulance also tested the system under various conditions (high speed driving, close to mountains, urban canyons, tunnels, etc). As the UMTS signal quality degrades below a certain threshold, the UE falls back to $2.5 \mathrm{G}$ (GPRS) and data rates are significantly reduced. A repeater was placed on top of the ambulance in order to enhance the signal levels thus maintaining the high bandwidth. The repeater is a simple amplifier, no bigger than a wireless access point, that picks up any available signal and boosts it up. Some forms of repeaters can work with an external antenna but most have the antenna embedded within their small structure. This was ideal to be placed on top of the ambulance, as it was very discreet and inherently weatherproof.

The system used the standard TCP/IP network to connect to the hospital, where the consultant resided. Several QoS parameters were monitored as the link was in progress: connection establishment delay, throughput, total delay, error rate, security, priority over other packets sent and resilience over unexpected loss of communication. In the higher OSI layers, the following parameters were collected: videoconferencing frame rate, achievable resolution, packets (and frames) dropped, sound quality.

\section{RESULTS}

For the four different scenarios described above, and by enabling or disabling the repeater on top of the ambulance, the following results were recorded:

\section{A. Best-case links}

Those links were found very close to base stations and further away from city centers so there will not be too much traffic demand. The highest speeds were achievable when the ambulance was cruising with speeds less than $20 \mathrm{Km} / \mathrm{h}$. The download speed in that case was a stable $1 \mathrm{Mbps}$ while the upload was about $300 \mathrm{Kbps}$. The maximum upload speed reached $570 \mathrm{Kbps}$ [Fig. 2]

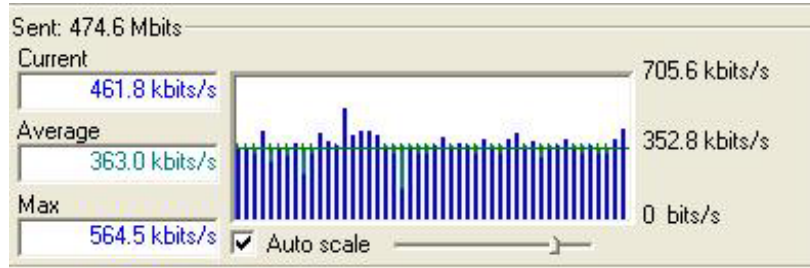

Figure 2. Uplink speed while videoconferencing (30 sec window)

The videoconferencing sessions were stable, no major delays were observed and for a resolution of 320x240 pixels 20 frames per second (fps) could easily be achieved. By increasing the resolution to $640 \times 480$ the frame rate dropped at about 6-9 fps.

\section{B. No HSPA links (fall back to $3 G$ )}

As the ambulance moved further away from the city, there was no provision for HSPA (it would be financially unviable to cover such large spaces with HSPA as currently, there is reduced demand for high data rates in those areas). The 
system initiated a fall back to standard UMTS (3G). The maximum speed of UMTS is about $384 \mathrm{Kbps}$. Practical measurements, however, indicated that the data rates did not exceed an average of $200 \mathrm{Kbps}$ download and $90 \mathrm{Kbps}$ upload. Fig. [3] shows the reduction in speed as the moving vehicle, cruising at a speed of $30 \mathrm{Km} / \mathrm{h}$ moves away from the HSPA coverage and into a UMTS area.

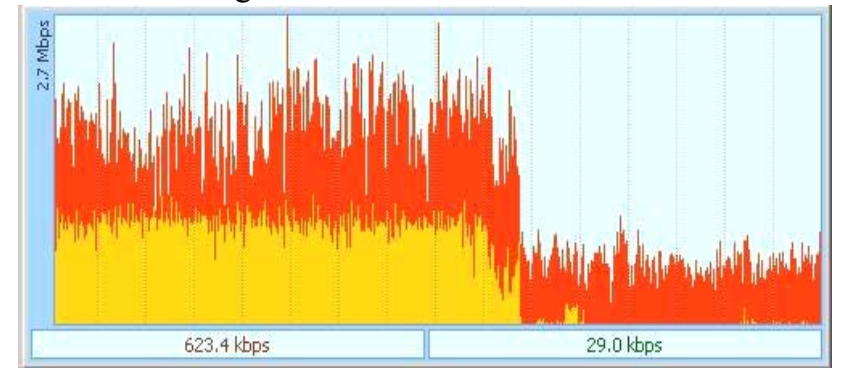

Figure 3. Traffic: fall back from HSPA (left) to standard UMTS (right) for a 15 minute window.

The videoconferencing sessions continued to work fine but at a reduced frame rate as less bandwidth was available. For the same $320 \times 240$ pixel resolution a frame rate of 4-8 fps was achieved. Frame fluctuation was relatively high and there was an increase on the delay to about $800 \mathrm{~ms}$.

\section{Congested areas (high data traffic)}

By keeping the ambulance in the city center, we forced it to use links that were already utilized by several other users. This is a more realistic approach as, in time, these networks will be used extensively. For this scenario, the data rates available were comparable with the non-congested links but had a much higher fluctuation both in bandwidth and in total delays. Bandwidth available averaged at 1 Mbps for downlink and $200 \mathrm{Kbps}$ uplink with delays varying between 200 and $1300 \mathrm{~ms}$. This resulted in the videoconferencing sessions to have varying quality (while the algorithm adjusted the compression ratio to fit the available bandwidth) and a frame rate ranging between 3 and 18 fps.

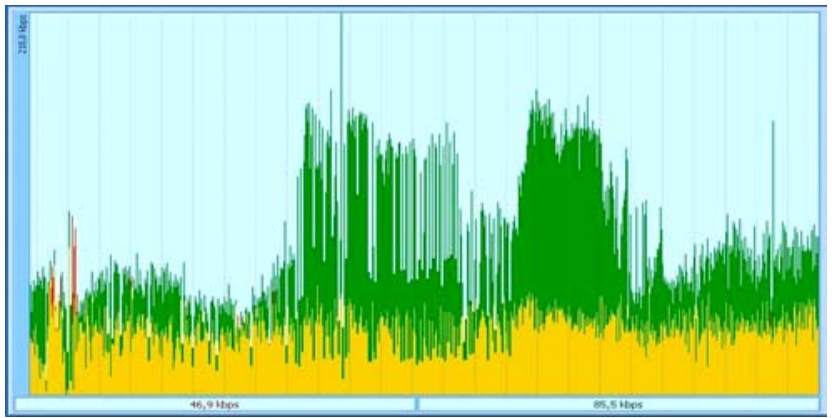

Figure 4. Bandwidth fluctuation in congested areas (15 min window).

\section{No $3 G$ coverage (fall back to GPRS)}

Either at a distance from the city center (where no 3G exists) or, most likely, under problematic conditions (tunnels, urban canyons, poor reception areas), the system falls back to GPRS communications. Ideally, GPRS should be able to provide about $128 \mathrm{Kbps}$ but practically it is only capable of handling less than $56 \mathrm{Kbps}$; most often less than that. No videoconferencing can be established then, as both the resolution and the frame rate was too low to be used for medical applications. High-resolution images can still be sent (pointing at patient's trauma or to ECG) but the time required for a $1 \mathrm{MB}$ image will be more than a minute.

As an additional scenario, a repeater was placed on top of the ambulance, and the same routes and procedures were followed. This increased the signal levels from about $-70 \mathrm{db}$ to about $-30 \mathrm{db}$ thus extending the HSPA area and significantly improving the system's performance.

\section{DISCUSSION}

The performance of the system was overall satisfactory and it was heavily depended on the location of the vehicle in relation to the base station as well as the congestion of that station.

Medical videoconferencing was of major concern. An average videoconferencing session requires 64-384 Kbps to be implemented effectively. In our case, the practical bandwidth exceeded the demand and was about $400 \mathrm{Kbps}$ uplink and 1.1 Mbps downlink in an average scenario (values reached at $570 \mathrm{Kbps}$ uplink and 5.6 Mbps downlink). The delays were also kept low, ranging between 300 and $1500 \mathrm{~ms}$ depending on the vehicle speed. The reduced delay also allowed for additional real-time applications to run from within the ambulance: Voice over IP, streaming video for tele-educating the paramedics, videocalling other ambulances, etc.

Still images were successfully sent using the same communication channel. Depending on their size (that ranged between 1 and $3 \mathrm{MB}$ ) they needed about 10 to $30 \mathrm{sec}$ to be transmitted. There was no significant degradation of video quality while the images were sent and while in oneway videoconferencing. For two-way video communication there was a relative reduction of frame rate from about 20 to $12 \mathrm{fps}$ and a small increase in delay, but nothing that could render the system unusable.

Sound was relatively clear with no significant break-ups. Sampling was kept at $8 \mathrm{KHz}$ yielding a nominal representation of frequencies between $0-4 \mathrm{KHz}$.

The use of the repeater significantly improved the system's performance. Depending on the route that the ambulance followed, a gain of 30 to $40 \mathrm{db}$ was observed. This translates to an increase of about 500 to $2000 \mathrm{Kbps}$ download and 50 to $300 \mathrm{Kbps}$ upload. Moreover, on threshold cases (urban canyons, low signal) that the system would have felled-back to GPRS, it maintained connection to HSDPA/HSUPA and avoided any gaps related with the switchover of the two systems.

Speed of the vehicle seemed to play an important role: while the ambulance speed remained less than $20 \mathrm{Km} / \mathrm{h}$ the 
delay was kept at 100-400 ms providing for a comfortable videoconferencing session. When the ambulance increased its speed to about $60-100 \mathrm{Km} / \mathrm{h}$ the delay increased to a maximum of $1.5 \mathrm{sec}$. There were two reasons for this: first of all, moving at high speeds meant that there would be a higher number of hand-overs to neighboring cells; especially $3 \mathrm{G}$ cells that were designed to have small radius in order to accommodate high traffic per area. Secondly, the HSPA protocols reduced the time transmission intervals (TTI) and thus the guard bands between those that ultimately dictate the maximum allowable window in time that a packet can be successfully received. This led the system to dynamically adapt more tolerating modulations that in turn resulted to an increase in delays.

The cost of the system ranged between 2000 and 3500 Euros, depending on the hardware used (laptop and camera used). The average cost of airtime is about 5 Euros per session, assuming that each session will last about 2-5 minutes and will use (collectively upload and download) about 20-50 MB of data. There are, however, package deals that allow for about $20 \mathrm{~GB}$ per month, with a modest cost of about 30-40 Euros.

When comparing this solution with its predecessor (UMTS) it is clear that even while using just HSDPA (asymmetric for higher downlink rates), the upload data rates significantly increased from a practical value of 64 $\mathrm{kbps}$ to about $600 \mathrm{kbps}$ [10]. It is clear, however, that upload speed is crucial as the main concern is high quality from the moving vehicle to the consultation point.

Overall, the results described above are in favor of using HSPA links for the purposes described. These links use better modulation and channelization techniques and are capable of offering a significant boost on the overall bandwidth available to the end user. This yields for a better quality of video (less compression needed thus less pixelization), faster still-image transferring and less delays.

The consultants that participated in this research were asked to evaluate the performance of the system based on the quality of the following criteria: video (involving factors like resolution, delay, frame rate, jitter, pixelization), audio (sound fidelity and audio/video delay), still image (resolution, color fidelity, performance using films and time to sent). Table 1 summarizes their opinions over the range of 25 trial runs involving human subjects carried by an ambulance. It reports the consultant's opinion on the percentage of data that were found to be of acceptable quality in order to be effectively used in delivering distant assistance.

TABLE 1

SUMMARY OF DOCTORS' FEEDBACK ON THE AUDIO / VIDEO QUALITY OF THE SYSTEM (\%)

\begin{tabular}{lcccc}
\hline $\begin{array}{l}\text { Quality (\%) vs. } \\
\text { scenarios }\end{array}$ & HSPA & $3 \mathrm{G}$ & $\begin{array}{c}\text { Congested } \\
\text { HSPA }\end{array}$ & $\begin{array}{c}\text { Fallback } \\
\text { to GPRS }\end{array}$ \\
\hline Video quality & $82 \%$ & $74 \%$ & $34 \%$ & $0 \%$ \\
Sound quality & $96 \%$ & $94 \%$ & $56 \%$ & $0 \%$ \\
Image quality & $94 \%$ & $90 \%$ & $82 \%$ & $44 \%$
\end{tabular}

The overall feeling of the individuals using the system (engineers, consultants and the paramedics) was that this is a very useful system producing high-quality results that can assist them in the performance of their duties when it was used in the ideal situation of best-case communications links. When the link fell back to $3 \mathrm{G}$ the situation was less than ideal but acceptable. In congested areas and over GPRS, some of the consultants felt that they would tire of the uncertainty of the image and the delay in reception.

\section{CONCLUSION}

In this paper we have presented a system capable of transmitting video, audio and images from a moving ambulance to a consultation point using HSDPA and HSUPA links. For a general acceptance of the system, there have to be ubiquitous presence of HSDPA and HSUPA implemented in areas where the ambulances operate. The system can easily be used by the paramedics and has reduced cost of installation and use. Results show that in most cases the system's outputs maintain high quality and can be used for a distant consultant to deliver expert opinion to the paramedics inside the ambulance. It is envisioned that widespread use of the system can assist a variety of emergency procedures and effectively save lives.

\section{REFERENCES}

[1] NHS trust, "London ambulance service, annual report 2006/07", London, 2007

[2] Pavlopoulos S., Kyriakou E., Berler A., Dembeyiotis, S., Koutsouris D. "A novel emergency telemedicine system based on wireless communication technology-AMBULANCE", Information Technology in Biomedicine, IEEE Transactions on Volume 2, Issue 4, Dec. 1998 Page(s): 261 - 267

[3] Konstantinos A. Banitsas, et. al., "Using 3G links to develop a teleconsultation system between a moving ambulance and an A\&E base station", International Journal of Telemedicine and Telecare, vol. 12, p. 23-25, Royal Society of Medicine press, 2006

[4] Yuechun Chu, Aura Ganz, "A mobile teletrauma system for rural trauma care", $26^{\text {th }}$ EMBC, San Francisco, 2004, p. 3282-3285

[5] Harri Holma, Antti Toskala, "HSDPA/HSUPA for UMTS: High Speed Radio Access for Mobile Communications", John Wiley \& Sons Ltd, 2006

[6] Cvitkovic, M.; Modlic, B.; Sisul, G., "High Speed Downlink Packet Access Principles", ELMAR, 2007, Sept. 2007 Page(s): 125 - 128

[7] Min Yang Jung, et.al., "Implementation of the Relay Server for the Ubiquitous Integrated Biotelemetry System for Emergency Care (UIBSEC) based on 3.5G HSDPA Technology", EMBS 2007. 29th Annual International Conference of the IEEE, 22-26 Aug. 2007 Page(s): $6713-6716$

[8] Jaemin Kang, et.al., "HSDPA (3.5G)-Based Ubiquitous Integrated Biotelemetry System for Emergency Care", EMBS 2007. 29th Annual International Conference of the IEEE, 22-26 Aug. 2007 Page(s): $3665-3668$

[9] Love, R.; Ghosh, A.; Weimin Xiao; Ratasuk, R., "Performance of 3GPP high speed downlink packet access (HSDPA)", Vehicular Technology Conference, 2004. VTC2004-Fall. 2004 IEEE $60^{\text {th }}$ Volume 5, 26-29 Sept. 2004 Page(s): 3359 - 3363 Vol. 5

[10] Pei Li; Weiling Wu, "The comparison of performances when WCDMA and HSDPA coexist in two different environments", Wireless Communications, Networking and Mobile Computing, 2005. Proceedings. 2005 International Conference on Volume 2, 2326 Sept. 2005 Page(s): $824-828$ 